Table S1: List of abbreviations

\title{
List of reactions
}

\begin{tabular}{|c|c|}
\hline Reaction & Catalyzing enzymes \\
\hline Acat1 & acetyl-Coenzyme A acetyltransferase 1 (acetoacetyl Coenzyme A thiolase) \\
\hline Acat2 & acetyl-Coenzyme A acetyltransferase 2 (acetoacetyl Coenzyme A thiolase) \\
\hline Aco1 & aconitase 1 , soluble \\
\hline Aco2 & aconitase 2 , mitochondrial \\
\hline Adh & $\begin{array}{l}\text { alcohol dehydrogenase IV } \\
\text { alcohol dehydrogenase V } \\
\text { alcohol dehydrogenase } 1 \mathrm{~A} \text { (class I), alpha polypeptide } \\
\text { alcohol dehydrogenase IB (class I), beta polypeptide } \\
\text { alcohol dehydrogenase 1C (class I), gamma polypeptide } \\
\text { alcohol dehydrogenase } 6 \text { (class V) } \\
\text { alcohol dehydrogenase } 7 \text { (class IV), mu or sigma polypeptide } \\
\text { alcohol dehydrogenase, iron containing, } 1 \text { (liver) } \\
\text { alcohol dehydrogenase } 1 \mathrm{AB} \\
\text { alcohol dehydrogenase } 1 \mathrm{AC}\end{array}$ \\
\hline Adh (con't) & alcohol dehydrogenase $1 \mathrm{BC}$ \\
\hline Adh5 & alcohol dehydrogenase $\mathrm{V}$ \\
\hline Akr & $\begin{array}{l}\text { aldo-keto reductase family } 1, \text { member A1 } \\
\text { aldo-keto reductase family } 1, \text { member B1 (aldose reductase) } \\
\text { aldo-keto reductase family } 7, \text { member A2 (aflatoxin aldehyde reductase) }\end{array}$ \\
\hline Akr1b1 & aldo-keto reductase family 1, member B1 (aldose reductase) \\
\hline Akr7a2 & aldo-keto reductase family 7, member A2 (aflatoxin aldehyde reductase) \\
\hline Aldh & $\begin{array}{l}\text { aldehyde dehydrogenase } 1 \text { family, member A1 } \\
\text { aldehyde dehydrogenase } 1 \text { family, member A2 } \\
\text { aldehyde dehydrogenase } 1 \text { family, member A3 } \\
\text { aldehyde dehydrogenase } 3 \text { family, member A1 } \\
\text { aldehyde dehydrogenase } 3 \text { family, member A2 } \\
\text { aldehyde dehydrogenase } 3 \text { family, member B1 } \\
\text { aldehyde dehydrogenase } 3 \text { family, member B2 } \\
\text { aldehyde dehydrogenase } 7 \text { family, member A1 } \\
\text { aldehyde dehydrogenase } 9 \text { family, member A1 }\end{array}$ \\
\hline Aqp9 & aquaporin 9 \\
\hline Bpgm & 2,3-bisphosphoglycerate mutase \\
\hline $\begin{array}{l}\text { Crns } \\
\text { (Carnitine } \\
\text { shuttle) }\end{array}$ & $\begin{array}{l}\text { Solute carrier family } 25 \text {, carnitine/acylcarnitine translocase, member } 20, \text { mitochondrial } \\
\text { carnitine acetyltransferase, mitochondrial }\end{array}$ \\
\hline Cyp2e1 & cytochrome $\mathrm{P} 450$, family 2 , subfamily E, polypeptide 1 \\
\hline
\end{tabular}




\begin{tabular}{|c|c|}
\hline Reaction & Catalyzing enzymes \\
\hline Fbp & $\begin{array}{l}\text { fructose-1,6-bisphosphatase } 1 \text { (liver) } \\
\text { fructose-1,6-bisphosphatase } 2 \text { (muscle) }\end{array}$ \\
\hline Flj32499 & hypothetical protein FLJ32499, mitochondrial \\
\hline Flj39207 & C219-reactive peptide \\
\hline Glo1 & glyoxalase I \\
\hline Grhpr & glyoxylate reductase/hydroxypyruvate reductase \\
\hline Hadha & $\begin{array}{l}\text { hydroxyacyl-Coenzyme A dehydrogenase/3-ketoacyl-Coenzyme A thiolase/enoyl-Coenzyme A hy- } \\
\text { dratase (trifunctional protein), alpha subunit } \\
\text { hydroxyacyl-Coenzyme A dehydrogenase/3-ketoacyl-Coenzyme A thiolase/enoyl-Coenzyme A hy- } \\
\text { dratase (trifunctional protein), beta subunit }\end{array}$ \\
\hline Hagh & $\begin{array}{l}\text { hydroxyacylglutathione hydrolase } \\
\text { hydroxyacylglutathione hydrolase-like }\end{array}$ \\
\hline Hmgcl & $\begin{array}{l}\text { 3-hydroxylmethyl-3-methylglutaryl-Coenzyme A lyase } \\
\text { 3-hydroxymethyl-3-methylglutaryl-Coenzyme A lyase-like 1, mitochondrial }\end{array}$ \\
\hline Hmgcs1 & 3-hydroxy-3-methylglutaryl-Coenzyme A synthase 1 (soluble) \\
\hline Hmgcs2 & 3-hydroxy-3-methylglutaryl-Coenzyme A synthase 2 (mitochondrial) \\
\hline Idh2 & isocitrate dehydrogenase 2 (NADP+), mitochondrial \\
\hline Idh1 & isocitrate dehydrogenase 1 (NADP+), soluble \\
\hline Ireb2 & iron-responsive element binding protein \\
\hline Ldh & $\begin{array}{l}\text { lactate dehydrogenase A (liver, skeletal muscle) } \\
\text { lactate dehydrogenase B (heart, red blood cell) } \\
\text { lactate dehydrogenase C (testis) } \\
\text { lactate dehydrogenase A-like 6B (testis) } \\
\text { lactate dehydrogenase A-like 6A } \\
\text { lactate dehydrogenase (heart, red blood cell, brain, kidney, liver, skeletal muscle) }\end{array}$ \\
\hline Ldha & lactate dehydrogenase A (liver, skeletal muscle) \\
\hline Ldhd & lactate dehydrogenase $\mathrm{D}$ \\
\hline Mdh1 & $\begin{array}{l}\text { malate dehydrogenase } 1 \text { (NAD), soluble } \\
\text { malate dehydrogenase } 1 \mathrm{~b}(\mathrm{NAD}) \text {, soluble }\end{array}$ \\
\hline Me1 & malic enzyme 1 , NADP(+)-dependent, cytosolic \\
\hline Oxct & $\begin{array}{l}\text { 3-oxoacid CoA transferase } 1 \text {, mitochondrial } \\
\text { 3-oxoacid CoA transferase 2, mitochondrial }\end{array}$ \\
\hline $\mathrm{Pc}$ & pyruvate carboxylase, mitochondrial (liver, kidney, intestine) \\
\hline Pck1 & phosphoenolpyruvate carboxykinase 1 , soluble (liver, kidney, intestine) \\
\hline Pck2 & phosphoenolpyruvate carboxykinase 2 , mitochondrial (liver, kidney, intestine) \\
\hline Pgam & $\begin{array}{l}\text { brain phosphoglycerate mutase } 1 \\
\text { muscle phosphoglycerate mutase } 2\end{array}$ \\
\hline Pgk & $\begin{array}{l}\text { phosphoglycerate kinase } 1 \\
\text { phosphoglycerate kinase } 2 \text { (testis) }\end{array}$ \\
\hline Slc16a1 & solute carrier family 16 (monocarboxylic acid transporters), member 1 \\
\hline
\end{tabular}




\begin{tabular}{|l|l|}
\hline Reaction & Catalyzing enzymes \\
\hline Slc25a1 & solute carrier family 25 (mitochondrial carrier; citrate transporter), member 1 \\
\hline Slc25a10 & solute carrier family 25 (dicarboxylate transporter) member 10, mitochondrial \\
\hline Uev & ubiquitin-conjugating enzyme E2-like \\
\hline Zadh & zinc binding alcohol dehydrogenase \\
\hline
\end{tabular}

List of reactions and catalyzing enzymes. In some cases several of the listed proteins are necessary to catalyze the reaction (for more information see the BiGG database, http://bigg.ucsd.edu).

\section{Metabolites}

\begin{tabular}{|c|c||c|c|}
\hline Abbreviation & Compound & Abbreviation & Compound \\
\hline $12 p p d-S$ & (S)-propane-1,2-diol & Lac-D & D-lactate \\
AaCoA & acetoacetyl-CoA & Lac-L & L-lactate \\
AcAc & acetoacetate & Lald-D & D-lactaldehyde \\
AcCoA & acetyl-CoA & Lald-L & L-lactaldehyde \\
CoA & coenzyme A & Lgt-S & (R)-S-lactoylglutathione \\
DHAP & dihydroxyacetone phosphate & Mal & Malate \\
FDP & fructose-1,6-bisphosphate & Mthgxl & methylglyoxal \\
FicytC & ferricytochrome c & OAA & Oxaloacetate \\
FocytC & ferrocytochrome c & OG & Oxoglutarate \\
G3P & Glyceraldehyde 3-phosphate & Pyr & pyruvate \\
G6P & glucose-6 phosphate & R5P & ribose-5-phosphate \\
Glc & glucose & Ru5P & ribulose-5-phosphate \\
Gthrd & reduced glutathione & Succ & succinate \\
HmgCoA & Hydroxymethylglutaryl-CoA & SuccCoA & Succinyl-CoA \\
Icit & Isocitrate & X5P & xylulose-5-phosphate \\
\hline
\end{tabular}

